\title{
Hexadactyly: A Rare Case
}

\author{
E Rossi $^{1}$, A Barbieri ${ }^{2}$, S Tamasi ${ }^{3}$, M Pignatiello ${ }^{2}$, MC Smaldone ${ }^{4}$, L Castelli ${ }^{1}$, S Cappabianca ${ }^{2}$ and M Zeccolini $^{1}$ \\ ${ }^{1}$ USD Radiology and Ultrasound, Santobono Pausilipon Hospital, Italy
}

${ }^{2}$ Medical and Surgical Department of Clinical and Experimental Intern, University of Naples, Italy

${ }^{3}$ UOC of General Radiology, Santobono Pausilipon Hospital, Italy

${ }^{4}$ Department of Advanced Biomedical Sciences, University of Naples Federico II, Italy

Received: September 15, 2017; Published: September 22, 2017

*Corresponding author: Eugenio Rossi, Department of Radiology and Ultrasound, Santobono Pausilipon Hospital, Posillipo Street, 226, Naples 80122,

Italy, Email: eugeniorossi3@libero.it

\section{Abstract}

A 2-year-old Caucasian female patient, referred to our Department, presented on physical examination a symmetrical duplication of the 5 th finger in both hands and feet. (Figure 1) Bone structures could be also palpated and observed at X-ray exam (Figures 1 \& 2). Clinical family history was negative for malformation disease or teratogenic drugs taken during pregnancy. Furthermore the child was not suffering from other associated malformations or organ dysfunction, the genetic examinations showed a normal karyo type and the absence of known mutations. She had no other anomalies and growth and development were normal. Radiographic examination showed six sets of phalanges with five metacarpals Figure 1; the last finger did not have an associated metacarpal in both hands but on the left the proximal phalanx of the last finger appears fused with the fifth metacarpal instead on the right hand the sixth finger is attached by a skin bridge only. The long bones of the arms were normal. According to Stelling and Turek classification, the right hand can be classificated as Type I, the left one as Type II [1].

Abbreviations: IFSSH: International Federation of Societies for Surgery of the Hand; ASSH American Society for Surgery of the Hand

\section{Introduction}

In 1964, Swanson proposed for the first time a classification system of congenital malformations of the hand. It was modified by the American Society for Surgery of the Hand (ASSH) and International Federation of Societies for Surgery of the Hand (IFSSH) in 1976. This classification includes congenital abnormalities in seven main categories: failure of formation, failure of differentiation, duplication, overgrowth, undergrowth, constriction band syndrome, and generalized syndromes and anomalies. Each category has subcategories based on anatomical, morphological or tissue type. With this system we can easily describe complex anomalies in a simple way [2]. A Type of duplication is polydactyly, this term introduced by Theodor Kerckring, is the development of more than five digits or toes [3].

A type of polydactyly is hexadactyly; it may be preaxial or radial/ tibial and post-axial or ulnar/fibular respectively. Polydactyly is one of the most common skeletal abnormalities [4]. In a Mexican retrospective case-control study the prevalence among 26,670 births was 1.73 in 1,000 live newborns. Instead in Chile, the prevalence has been estimated as 1.33 in 1,000 live newborns [5]. We describe a rare case of sporadic familial postaxial hexadactyly of all four limbs (Figure 1).

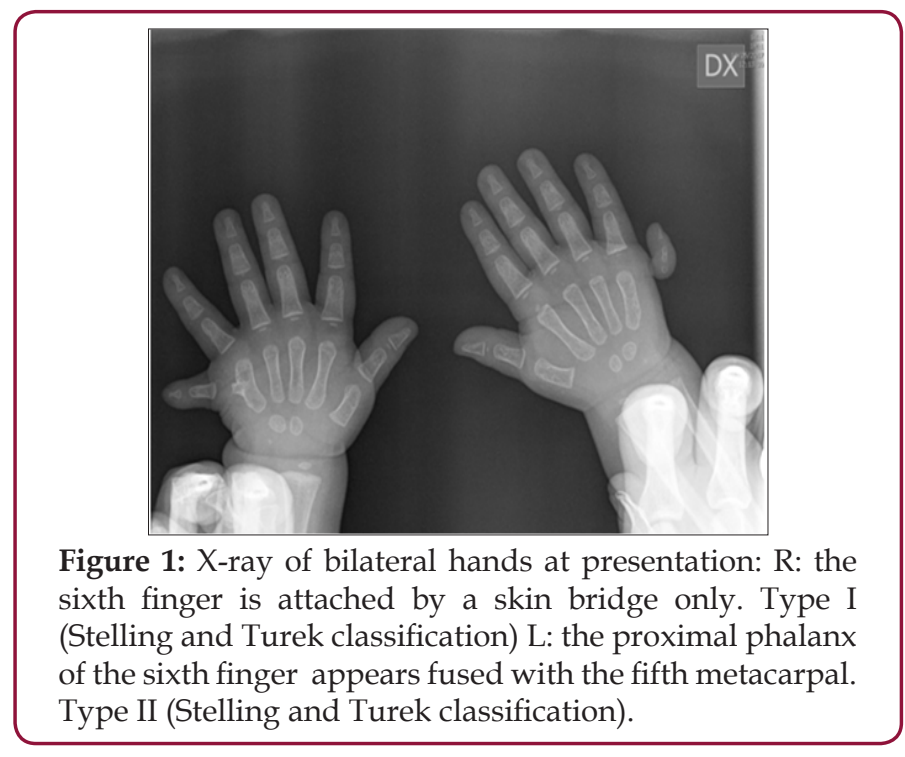

\section{Results/Observation}

Polydactyly of the hand, like polydactyly of the foot, has manifolds morphologic phenotypes. The surgical methods are diverse and based on the subtype of the finger or toe [2], the 
prognosis varies greatly [5]. Rarely have been described in the literature a hexadactyly of all four arts. Our clinical case is very particular because the child is Caucasian and female, there isn't familiarity, any co-morbidities and her karyo type is normal. Another peculiarity is the differerent type of post-axial polydattily for both the hand and the feet. These conditions make this case very interesting and absolutely exclusive. The performed analysis of the literature confirmed the peculiarity of the associated features of this case (Figure 2).

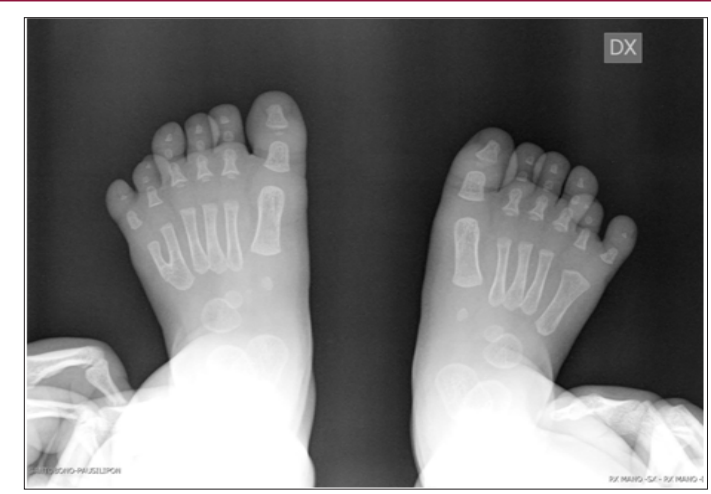

Figure 2: X-ray of bilateral feet at presentation: R: six sets of phalanges with five metatarsals, the fifth seems to be generated by the fusion of the fifth and sixth metatarsals. L: six sets of phalanges with six metatarsals, the fifth and the sixth appear proximally fused.

\section{Discussion}

Post-axial polydactyly of the hand is most frequent specially in Africans and Afro-Americans, the incidence is one in 143 live births, exactly 10 times greater than the incidence compared to their Caucasian counterparts: one in 1,339 live births [6]. When present in Caucasians, post-axial polydactyly is associated with an autosomal recessive transmission and syndromic conditions such as Ellis-van Creveld and chondroectodermal dysplasia [7]. This malformation of the hand is associated with African Black ethnicity, male sex, twinning, parental consanguinity, and there is frequent recurrence in 1st-degree relatives. Instead polydactyly of foot is associated with Amerindian racial background, parental sub fertility, and bleeding in the 1st trimester of pregnancy. Bilateral form displayed the highest frequency of associated congenital defects. [4] These deformities can occur in many varieties, from a simple extension of a distal phalanx, involving carpal or tarsal bones, to a simple or multiple duplications [8].

Stelling (1963) and Turek (1967) proposed a classification in order to describe post-axial polydactyly, subdividing it into three groups according to the morphological appearance:

a) Type I extra digit attached by skin bridge only;

b) Type II extra digit articulating with metacarpal or phalanx;

c) Type III extra digit articulating with extra metacarpal;

Recently Seok HH has introduced a new classification system of polydactyly of the foot named "SAM." (Table 1) The fundamental principle is to classify polydactyly according to three categories. Each category is also subdivided into three groups. Theoretically, 27 permutations $(3 \times 3 \times 3)$, or classes, are possible. Each category has its own meaning. The category of syndactylism (S) reflects the need for skin grafting during surgery. Syndactylism of the adjacent toe, to be retained, determines the $\mathrm{S}$ category. Through the syndactylism grouping $\left(\mathrm{S}_{0}\right.$ to $\left.\mathrm{S}_{2}\right)$, we expect to anticipate the surgical time. Axis deflection of the toe to be save $(A)$ reflects the need for wedge ostectomy during surgery $\left(A_{0}\right.$ to $\left.A_{2}\right)$. The category of metatarsal extension (M) is an important component in the description of morphology and determines the type of surgical approach to the duplicated bone. The metatarsal bone of the foot plays a key role in supporting the transverse arch of the foot, which primary function is to support the body. In $M_{1}$ to $M_{2}$ cases, we cannot rule out the possibility of postoperative residual bone deformity or angulation deformity [9].

Table 1: New classification system of polydactyly of the foot named "SAM".

\begin{tabular}{|c|c|c|c|}
\hline Sam Classification & S Syndactylism to the adjacent toec & A Angulation/Deviation & M Metatarsal extension \\
\hline 0 & No & $<15^{\circ}$ & No \\
\hline 1 & Incomplete, $<1 / 2$ & $15^{\circ} \leq<30^{\circ}$ & Metatarsal head shared, but shaft (-) \\
\hline 2 & $1 / 2 \leq \leq$ complete Complex syndactyly & $\geq 30^{\circ}$ & Shaft $(+)$ and more \\
\hline
\end{tabular}

\section{References}

1. Uwe Wollina, Shyam B (2010) Verma Sporadic familial ulnar hexadactyly of all four limbs. J Dermatol Case Rep 11: 4(1): 6-10.

2. Guo B, Lee SK, Paksima N (2013) Polydactyly: a review. Bull Hosp Jt Dis 71(1): 17-23.

3. Gawlikowska-Sroka A, Tudaj W, Czerwiński F (2009) A and B preaxial polydactyly with syndactyly of feet and hands in the same person-a case report. Adv Med Sci 54(2): 305-307.

4. Castilla EE, Da Graca Dutra M, Lugarinho da Fonseca R, Paz JE (1987) Hand and foot postaxial polydactyly: two different traits. J Hand Surg Am 12(3): 474-476.

5. Hyo Hyun Seok, Ji Ung Park, Sung Tack Kwon (2013) New Classification of Polydactyly of the Foot on the Basis of Syndactylism, Axis Deviation, and Metatarsal Extent of Extra Digit. Arch Plast Surg 40(3): 232-237.
6. W Blauth, AT Olason (1988) Classification of Polydactyly of the Hands and Feet. Arch Orthop Trauma Surg 107(6): 334-344.

7. Villa MC, Rocca A, Kantar A, Lovati C, Porcelli F (2006) Hexadactylism of the four limbs: a case report. Pediatr Med Chir 28(4-6): 101-105.

8. Hyo Hyun Seok, Ji Ung Park, Sung Tack Kwon (2013) New Classification of Polydactyly of the Foot on the Basis of Syndactylism, Axis Deviation, and Metatarsal Extent of Extra Digit. Arch Plast Surg 40(3): 232-237.

9. Mai P Nguyen, Ericka A Lawler, Jose A Morcuende (2014) A Case Report of Bilateral Mirror Clubfeet and Bilateral Hand Polydactyly. Iowa Orthop J 34: 171-174. 


- Assets of Publishing with us
- Immabal archiving of articles

